\title{
Verschwörungsglaube in der Pandemie
}

\author{
Volker Weiß
}

Online publiziert: 8 . Dezember 2021

(C) The Author(s), under exclusive licence to Springer Fachmedien Wiesbaden GmbH, ein Teil von Springer Nature 2021

Zusammenfassung Der Vortrag führt in verschwörungstheoretische Konstruktionen ein, die im Zuge der Proteste gegen die Anti-Corona-Maßnahmen (nicht nur) in Deutschland Aufmerksamkeit bekommen haben. Dabei steht besonders die Legende eines „Great Reset“ im Mittelpunkt, anhand derer das Protestmilieu ein Programm des Weltwirtschaftsforums zur Krisenbewältigung zum Nachweis einer künstlichen „Plandemie“ umdeutet. In diesem vor allem von neurechter Seite beschworenen Szenario soll durch eine angeblich die Finanzmärkte beherrschende ,globalistische Elite“ der Kommunismus eingeführt werden; eine Verschwörungstheorie, die nicht zufällig an antisemitische Legenden der Vergangenheit erinnert. Die Wiederkehr solch traditioneller Semantiken während der Corona-Pandemie bestätigt einmal mehr die Langlebigkeit paranoider und autoritärer Weltanschauungen.

Schlüsselwörter Corona-Pandemie · Verschwörungstheorie · Verschwörungsideologie · „Querdenker“ · Neue Rechte · Corona-Proteste · Antisemitismus

Bei diesem Text handelt es sich um das Manuskript eines Vortrags, den Volker Weiß am 08.11.2021 in der Reihe „Andere als Gefahr - die Gefährdung des Anderen. Die Pandemie als gesellschaftliches Problem“"gehalten hat. Die Reihe wird im Winter 2021/22 von der DGS-Sektion Soziale Probleme und soziale Kontrolle ausgerichtet.

Volker Weiß $(\bowtie)$

Zentrum für Antisemitismusforschung (ZfA), Fakultät 1, Freie Universität Berlin,

Kaiserin-Augusta-Allee 104-106, 10553 Berlin, Deutschland

E-Mail: Volkerweiss-hh@gmx.net 


\title{
Conspiracy Belief and the Pandemic
}

\begin{abstract}
The lecture introduces constructions of conspiracy theories that have received attention (not only) in Germany in the course of the protests against the anticorona measures. The focus is particularly on the legend around the "Great Reset", a program of the World Economic Forum for crisis management that is reinterpreted by the protest milieu to prove an artificial "plandemy". In this scenario, invoked primarily by the new right, a "globalist elite" allegedly ruling the financial markets is said to introduce communism. Not accidentally, this is a conspiracy theory that reminds of antisemitic legends of the past. The return of these traditional semantics during the Corona pandemic once again confirms the longevity of paranoid and authoritarian worldviews.
\end{abstract}

Keywords Corona pandemic $\cdot$ Conspiracy theory $\cdot$ Conspiracy ideology · "Querdenker" · New Right · Corona protests · Antisemitism

Bevor ich mich der gängigen verschwörungstheoretischen Deutung der Covid-19Pandemie zuwende, möchte ich einige Anmerkungen zur konspirationistischen Weltsicht allgemein vorausschicken. Zunächst: Selbstverständlich gibt es Verschwörungen, also geheime Absprachen zwischen verschiedenen Akteuren zur Schädigung anderer und Durchsetzung eigener Ziele. Wenn sich z. B. die führenden Autohersteller untereinander absprechen, um den Preis für Stahl zu drücken, könnte man das eine Verschwörung nennen. Auf jeden Fall ist es ein Fall fürs Kartellamt. Wenn sich eine Regierung mit der Generalität und der Industrie im Geheimen darauf verständigt, binnen kurzer Zeit aufzurüsten, um den Nachbarstaat militärisch anzugreifen, könnte man das eine Verschwörung nennen - oder Planung eines Angriffskrieges. All das gibt es - im Geschäftsleben, in der Politik und anderswo. Bei der Aufdeckung solcher Dinge ist es nützlich, nach Mustern zu suchen. Es lassen sich Verschwörungshypothesen aufstellen und überprüfen. Mitunter endet die Sache vor Gericht, waren die Hypothesen nicht haltbar, löst sich möglicherweise alles in Luft auf. Manche Menschen können jedoch nicht davon lassen, überall nach Mustern zu suchen und werden irrational. Dann spricht man umgangssprachlich von Verschwörungstheorien, neuerdings - um den Theoriebegriff zu retten - auch von Verschwörungsideologie, Verschwörungsglauben, Verschwörungserzählungen u. ä. Um dieses Phänomen im Kontext der Corona-Pandemie soll es hier gehen. (Vgl. weiterführend Kleffner/Meisner 2021, Speit 2021, Amadeu Antonio Stiftung 2021 und neuerdings Benz 2021)

Michael Butter (2018, S. 34) unterscheidet in seiner grundlegenden Arbeit über Verschwörungstheorien zwischen drei Typen:

1. Die Ereignisverschwörungstheorie: Sie geht davon aus, dass ein bestimmtes Geschehen Ergebnis eines Komplotts war (Kennedy-Mord, Mondlandung, 11. September). 
2. Die Systemverschwörungstheorie: Sie geht davon aus, dass bestimmte Gruppen „für eine ganze Reihe von Ereignissen verantwortlich“ sind, um die Macht zu erringen oder zu erhalten (Freimaurer, der Vatikan, Juden, Kommunisten, etc.).

3. Die Superverschwörungstheorie, in der sich Ereignis- und Systemverschwörungstheorie kombiniert finden. Kurz gesagt: Darin sind praktisch immer die gleichen Akteure für alle schädlichen Ereignisse verantwortlich.

Michael Butter erinnert daran, dass das Phänomen des Verschwörungsglaubens keineswegs neu ist. Es ist seit Jahrhunderten präsent, früher waren Verschwörungstheorien sogar als herrschaftskritisches „Wissen“ etabliert. Neu hingegen war die Problematisierung, als nach dem Zweiten Weltkrieg eine systematische Hinterfragung verschwörungstheoretischer Erzählungen begann.

Ich denke, dass es dafür mehrere Gründe gibt: So sind die modernen Gesellschaften in vielen Belangen wesentlich transparenter geworden als noch die Staaten des 19. Jahrhunderts. Auch in den bürgerlichen Republiken war Politik noch lange eine elitäre Angelegenheit unter Ausschluss der Öffentlichkeit. Das öffnete nicht nur allerlei Absprachen Tür und Tor, sondern lud zu Spekulationen über solche Szenarien ein. Gesellschaften, deren politische Struktur von korrupten „Rackets“ gekennzeichnet ist, haben wohl bis heute eine Vorliebe für solche Narrative, wie beispielsweise ein Blick in den Nahen Osten zeigt. ${ }^{1}$ Das führt auch zur Frage, was ihr Aufschwung der letzten Jahre auch in der westlichen Welt eigentlich über den Zustand der Gesellschaften aussagt.

Wie erwähnt galten im Westen Verschwörungstheorien in den letzten Jahrzehnten aus guten Gründen als anrüchig. Mitte des 20. Jahrhunderts hatte die Shoah gezeigt, wohin antisemitische Legenden führen können, die ja eine deutlich konspirationistische Struktur aufweisen. Es waren vor allem Autoren der Kritischen Theorie wie Leo Löwenthal und Theodor W. Adorno, die den Zusammenhang von Verschwörungsmotiven mit autoritärer Subjektdisposition und totalitärer Herrschaft untersucht haben. Ich fürchte, Verschwörungsglaube lässt sich in gewisser Weise auch als eine Art verkümmerte Herrschaftssoziologie lesen, mittels der die Erfahrung gesellschaftlicher Schieflagen in paranoide Spekulationen abgebogen wird. Paradoxerweise stützt diese Form der Rebellion die Missstände eher als sie anzugreifen. Hier ist offensichtlich viel Wissen verloren gegangen, wie die jüngste Renaissance des Themas zeigt. Die Beratungsstelle Sekten Info Nordrhein-Westfalen teilt beispielsweise in ihrer Jahresauswertung 2020 mit, dass sich ,,[b]esonders in der Kategorie ,Psychogruppen“, in der die vielen Anfragen zu Verschwörungstheorien gezählt wurden, ... die Anfragen verfünffacht" haben. ${ }^{2}$ (Vgl. hinsichtlich dieses Phänomens auch Ministerium des In-

\footnotetext{
${ }^{1}$ Der Begriff „Racket“, ursprünglich eine amerikanische Slang-Bezeichnung für organisierte Kriminalität, wurde ab den Vierzigerjahren seitens des Frankfurter Instituts für Sozialforschung für herrschaftskritische Überlegungen verwendet. Er sollte den Verfall der Kategorie der Marx’schen „Klasse“ zur bandenartigen Organisation von Interessengruppen innerhalb der bürgerlichen Gesellschaft kennzeichnen. Bis heute wird er polemisch auf Gesellschaften angewandt, in denen Interessengruppen den wirtschaftlichen und staatlichen Bereich mit korrupten Strukturen durchzogen und (wie z. B. im Libanon) bis zur Dysfunktionalität unter sich aufgeteilt haben.

2 https://sekten-info-nrw.de/information/zahlen-und-fakten. Zugegriffen: 4. November 2021.
} 
neren des Landes Nordrhein-Westfalen 2021 und Österreichische Bundesstelle für Sektenfragen 2021.)

Dass die Verschwörungserzählungen nun aus den Randbereichen der politischen Exzentrik und der Unterhaltungsindustrie (kein Thriller ohne guten Verschwörungsplot!) wieder massiv in die Politik zurückgekehrt sind, dürfte mit dem Aufschwung des Populismus zu tun haben. Es gibt, wie Butter (2018, S. 17) betont, ,strukturelle Parallelen zwischen populistischen und konspirationistischen Argumentationsweisen“. Hinzu kommt eine digitale Kommunikationsstruktur, die der schnellen und globalen Verbreitung von Nachrichten und Gerüchten entgegenkommt. Ein Blick in die US-Politik der jüngsten Vergangenheit zeigt, dass Verschwörungsnarrative oft genug ein probates Mittel zur Mobilisierung von erregten Massen sind.

Samuel Salzborn (2021, S. 41) merkt an, dass eine Pandemie ,ein geradezu idealtypischer Vorwand für Verschwörungsmythen“ ist. Wie sehr Krankheiten und Epidemien Verschwörungserzählungen angeregt haben, zeigte sich von den Pestlegenden bis hin zu den Theorien über eine angebliche Herkunft des AIDS-Erregers aus einem CIA-Programm. Es gab zudem bereits vor Corona einen Aufschwung solcher Legenden. So bildete sich um die Anschläge vom 11. September 2001 eine ganze Szene, die unter dem Namen „Truther“ eine Urheberschaft der USA an den Anschlägen nachweisen wollte. Dass sich dies unabhängig von den politischen Lagern verbreitete, zeigte der erste deutsche Bestseller, der mit dieser These hausieren ging. Er stammte von dem Taz-Autor Matthias Bröckers (Bröckers 2002).

Analog dazu rankten sich unter dem Begriff ,Birther“" Legenden um den angeblich ausländischen Geburtsort von Barack Obama, um dessen Präsidentschaft zu delegitimieren. Während der Trump-Administration machte unter dem Namen ,QAnon" die Behauptung weltweit Karriere, internationale Finanzeliten betrieben pädophile satanistische Zirkel zur Beherrschung der Menschheit. Donald Trump führe einen versteckten Krieg gegen diese Machenschaften und würde deshalb vom liberalen (also „kulturmarxistischen“) Establishment bekämpft. Hier kann man leicht Strukturelemente identifizieren, die bereits in Verschwörungserzählungen vorheriger Jahrhunderte, etwa als antisemitische Ritualmordlegenden, kursierten. Q-Anon trug wesentlich zur Radikalisierung der Anhänger Trumps (auch in Deutschland) bei.

Nach Trumps Niederlage wurde die Behauptung von einer durch Manipulation „gestohlenen Wahl“ in die Welt gesetzt. Darauf aufbauend gab es hierzulande vor der Bundestagswahl einige zaghafte Versuche der AfD, die Briefwahl zu diskreditieren.

Angesichts dieser Renaissance von Verschwörungsnarrativen der letzten Jahre ist es kaum verwunderlich, dass auch die Corona-Pandemie umgehend zum Gegenstand entsprechender Deutungen wurde. Schließlich befindet sich die liberale Demokratie westlichen Zuschnitts in einer Krise. Schon eine erste Auswertung der ,politischen Soziologie der Corona-Proteste“ durch die Universität Basel in Zusammenarbeit mit dem Institut für Protest- und Bewegungsforschung vom Dezember 2020 sieht die Querdenker-Strömung als „Ausdruck einer fundamentalen Legitimationskrise der modernen Gesellschaft“" (Institut für Soziologie der Universität Basel 2020, S. 62). Die Vorformen der Proteste in Deutschland, Österreich und der Schweiz werden in inhaltlich sehr unterschiedlichen, aber formal ähnlich agierenden außerparlamentarischen Protestbewegungen sowohl rechter als auch linker Provenienz ausgemacht. 
Hinsichtlich der etablierten gesellschaftlichen Prozesse demokratischer Teilhabe gelten sie durchaus als problematische Entwicklung:

Unsere Beobachtungen lassen den Schluss zu, dass das Verhältnis [der Teilnehmenden; V.W.] zu etablierten Institutionen wie den Staat, die Medien oder die Wissenschaft als insgesamt ambivalent bis hin zu entfremdet bezeichnet werden kann (ebd.: 56).

Neben der Absage an den rationalistischen Grundkonsens der Hypermoderne, die mit einer Rückwendung zu esoterisch-spiritualistischen Weltbildern einhergeht, hat dieser Vorgang auch politische Auswirkungen, stellen die Autor:innen der Basler Studie fest:

Die Bewegung der Querdenker:innen ist vor allem durch eine tiefe Entfremdung von Kerninstitutionen der liberalen Demokratie zu charakterisieren. Der parlamentarischen Politik und den Parteien, der Wissenschaft und den Medien - allen Institutionen schlägt grosses Misstrauen entgegen (ebd.: 62).

Nun ist grundsätzliches Misstrauen gegenüber Institutionen nichts per se Schlechtes, angesichts zugleich hoher Werte für verschwörungsideologische Ansätze unter den Befragten ist hier jedoch Skepsis angebracht. So erreicht die Behauptung einer Steuerung politischer Prozesse durch ,geheime Organisationen“ eine Zustimmung von $50 \%$, etwas mehr noch sehen Politiker hauptsächlich als ,Marionette dahinterstehender Mächte“ (ebd.: 21).

Mit etwas mehr Zeit und breiterer Quellenbasis hat die Leipziger Autoritarismusstudie „Alte Ressentiments, neue Radikalität“ diese ersten Basler Befunde zu den Corona-Protesten bestätigen und vertiefen können. Auch sie sieht die demokratische Kultur durch Verschwörungstheorien herausgefordert und widmet dem Thema „Aberglaube, Esoterik und Verschwörungsmentalität in Zeiten der Pandemie“ ein eigenes Kapitel. Nach ihrem Befund dient Verschwörungsmentalität als „Kitt“ einer rechts-links-Querfront seit den Montagsmahnwachen (Decker und Brähler 2020, S. 283).

Die Leipziger Studie ermittelte unter den Protestierenden zudem hohe Zustimmungswerte in den Bereichen Verschwörungsglaube, Esoterik und Aberglaube. „Diese Fusionierung ist“, wie die Autor:innen feststellen, ,weder neu noch überraschend", immerhin ließen sich

,zahlreiche Verbindungslinien von Verschwörungsmentalität und Aberglauben bzw. Esoterik finden: Die drei geteilten Grundüberzeugungen ,Nichts passiert durch Zufall', ,Nichts ist wie es scheint" und ,Alles ist miteinander verbunden “ ... fassen die Gemeinsamkeiten von Verschwörungsmentalität und esoterischem Aberglauben auf einer beschreibenden Ebene gut zusammen“ (ebd.: 292).

In der Corona-Pandemie hat diese Haltung zu einer speziellen Verschwörungserzählung geführt, die ich hier kurz vorstellen möchte: dem „Great Reset“.

Unter „Great Reset“" verstehen die Anhänger der Legende einen Masterplan globaler Eliten zur Unterjochung der Welt, in dem der Corona-Pandemie die Rolle des Beschleunigers zukommt. Demnach ginge es bei den Maßnahmen zur Eindäm- 
mung der Virus-Epidemie um nichts Geringeres als die Errichtung einer Diktatur, um mögliche Widerstände auszuschalten.

Gerade seitens Neuer Rechter und Identitärer Bewegung wurde mit der Legende vom „Great Reset“ nahtlos an das vorher dominante Narrativ vom „Großen Austausch“ angeknüpft.

Das zeigt sich auch bei der identitären Aktivistin Caroline Sommerfeld, Autorin der Zeitschrift Sezession aus dem neurechten Antaios Verlag. Unter Berufung auf amerikanische ,Truther“ meint sie, an der Pandemie Elemente einer „Silent Weapon“ des US-Militärs zum besseren ,social engineering“ zu erkennen. Es sei ein gigantisches „Manipulations- und Planspiel“ im Gange (Sommerfeld 2021).

Die Rede vom „Great Reset“ folgt der durch Michael Butter beschriebenen Grundstruktur verschwörungstheoretischer Erzählungen, „Geschichte immer vom Ende her“ zu erzählen (Butter 2018, S. 59). Ursprünglich waren unter dem Begriff Reformvorschläge des Weltwirtschaftsforums zur Krisenbewältigung gebündelt. Verschwörungstheoretisch gekapert und in Verkehrung von Ursache und Wirkung wurde er nun jedoch als Beleg dafür präsentiert, dass die Pandemie geplant gewesen sei - eine „Plandemie“. Dieser Uminterpretation zufolge dient der „Great Reset", kaschiert als Kampf gegen Covid19 und den Klimawandel, lediglich der Einführung eines neuen Sozialismus.

Vor einem „Seuchensozialismus“ warnt beispielsweise ein Bericht der AfD-nahen Jungen Freiheit. Der Zeitung zufolge hatte u. a. die ehemalige DDR-Bürgerrechtlerin Vera Lengsfeld auf einer Konferenz die These präsentiert, dass „Hinter der Corona-Maske ... klammheimlich der ,Great Reset' (,Große Transformation“) ins Werk gesetzt" werde (Dorn 2020).

Auch die Verwendung des Schlagworts „Great Reset“ durch die AfD zeigt, dass der Glaube an eine gesteuerte Pandemie eine gewisse Verbreitung hat. Bemerkenswert ist dabei, dass seine angeblichen Akteure zugleich als Agenten kapitalistischer Globalisierung und als „Kommunisten“ gesehen werden. Da es sich hierbei zudem um historische Semantiken aus dem Fundus des Antisemitismus handelt, ist es nur konsequent, dass sich die Agitation mit Georg Soros auch gegen einen jüdischen Akteur richtet.

Das bleibt nicht nur auf die Kreise der äußersten Rechten beschränkt. Im weiteren Milieu der Corona-Proteste macht man ebenfalls einen Zusammenhang zwischen der Pandemie und einem Masterplan zum „Great Reset“ aus. ${ }^{3}$ In dieser Lesart steht Covid 19 für die Agonie des globalen Systems. In der Querdenker-Zeitschrift Demokratischer Widerstand ist man davon überzeugt, dass im Schatten von Corona gezielt die alte Ordnung abgeschafft werde und formuliert plakativ: „Die transnationale Elite des Globalkapitalismus führt derzeit Krieg gegen die Zivilgesellschaft“ (Mies 2021).

Die Pandemie wird so als Endkampf um die Zukunft gedeutet, bei dem alle verfügbaren Mittel zum Einsatz kämen. Angesichts des zunehmenden Widerstandes

\footnotetext{
3 Vgl. Aya Veláquez, „China und der Great Reset“, in: Demokratischer Widerstand 28/2020, S. 12-15 und „Die Agenda ,The Great Reset“ wird unser Leben komplett verändern - China als Vorbild!“‘. Tube Querdenken 711. https://tube.querdenken-711.de/videos/watch/b3ff0e1b-aee0-44f4-93ea-a140ae49501a. Zugegriffen: 4. November 2021.
} 
seien die vornehmlich in den USA beheimateten, aber weltumspannend agierenden Eliten gezwungen, zur Aufrechterhaltung ihres Systems die Realität zu verzerren. Michael Ballweg, Gründer der Querdenker-Bewegung, bringt dort im Interview ebenfalls das Motiv des „Great Reset“ ins Spiel (Ballweg 2021).

In der Zeitschrift Sezession zählt Thomas Hoof Faktoren und Profiteure des europäischen Niedergangs auf. Ihm zufolge gehe mit Corona und „Great Reset“ die Entmachtung der europäischen Industrienationen in die letzte Phase über. Die Leidtragenden dieses Wandels, die souveränen Staaten und ihre Nationalökonomien, würden durch Ökologie- und Humanitätsrhetorik sowie mittels Angstkampagnen in Schach gehalten. Das globale Finanzkapital habe zur Zerschlagung der nationalen Realwirtschaft eine „fiktionale Epidemie“ (Covid 19), die „fiktionale Demokratie“ (Wahlniederlage Trumps) und die ,fiktionale CO2-Erwärmung“ (Klimawandel) geschaffen. Entgegen seiner kapitalismuskritischen Andeutungen bietet Hoof jedoch keine sozioökonomische Analyse des Problems der Kapitalkonzentration, sondern schwenkt auf eine Personalisierung um. Er entwickelt die Vorstellung eines transnationalen Netzwerks, das als „Ordnungszentrum“ weitgehend die Prozesse steuere und vor allem die Kapitalabschöpfung monopolisiere. Dessen Struktur werde dabei allerdings nur oberflächlich sichtbar. Tatsächlich, so raunt sein Text, sei über dieses Zentrum ,wenig bekannt, es sei denn, man traute den alten verschwörungstheoretischen Quellen“. Hoof bewegt sich innerhalb des klassisch antisemitischen Schemas vom ,schaffenden“ und ,raffenden“ Kapital. Die von ihm vermuteten globalen Akteure erklärt er in Umkehrung des Begriffs „,Verschwörungstheoretiker“ zu „Verschwörungspraktiker[n]“" und bedient mit Blick auf die Abwahl Donald Trumps die klassische Erzählung einer langfristigen Konspiration:

Nach mehr als 200 Jahren Vorbereitung und einer wirklich beeindruckenden Planungs- und Handlungstreue über Generationen hinweg, gestärkt von immer frischem Trank aus tiefen freimaurerischen, sektenpuritanischen und talmudischen Weisheitsquellen, kurz vor Schluß dann nur dies: gefälschte Wahlzettel (Hoof 2021).

Bemerkenswert ist, dass die politische Entwicklung Hoofs der Gemengelage in den Corona-Protesten heute nicht unähnlich ist. Reste konsumkritischer Terminologie und Anleihen bei linker Globalisierungskritik zeugen davon. Hoof hatte sich nach seinen Anfängen bei den Grünen und als Gründer von Manufactum, einer Handelskette für nachhaltige Produkte, schließlich der Neuen Rechten angeschlossen. Heute ist sein Hauptbetätigungsfeld die ehemals Manufactum angegliederte Verlagsbuchhandlung Manuscriptum.

Auf Hoofs Leserschaft zielt auch die Zeitschrift Tumult, deren Werkreihe von Manuscriptum vertrieben wird. Das Manuscriptum Verlagsprogramm wird in $T u$ mult mit dem Slogan „Ohne Mundschutz im Kopf“ beworben. ${ }^{4}$ Angesichts dieser Verbindung ist es nicht überraschend, dass in Tumult eine ähnliche Sicht auf die Pandemie vertreten wird. Peter J. Brenner bilanzierte dort das ,staatliche CoronaRegime [als] eine Kriegserklärung an die bürgerliche Gesellschaft"“. Brenner ruft ebenfalls das Motiv eines „Great Reset“ unter Zerstörung von Geschichte und Iden-

\footnotetext{
4 Vgl. Tumult, 2/2021, S. 113.
} 
tität auf: „Das Corona-Regime fügt sich ein in den Gesamtkomplex einer Tabularasa-Politik, die aus der bürgerlichen Gesellschaft ein leeres Blatt macht, das sich neu beschreiben lässt.“ (Brenner 2021)

Ein anderer Autor von Tumult präsentiert mit Rückgriff auf Oswald Spengler Corona als einen Übergang in eine kommunistisch-technofuturistisch geprägte Dystopie. Dabei blättert er in sämtlichen Enteignungsängsten, die aus dem Diskurs des Rechtspopulismus bekannt sind:

Der postpandemische Mensch wird nichts mehr besitzen, aber glücklich sein, wie die Planspiele der Großen Transformation und die Glücksversprechen des Great Reset verlauten lassen; er hängt nicht mehr am alten Modus der Verdinglichung und spürt nicht mehr den alten analogen Impuls als Eigentümer und Besitzer, Goldsparer oder Bargeldzahler, gegenständlichen Objektivitäten verhaftet zu sein (Kießling 2021).

Dies waren einige Beispiele für die Verbreitung von Verschwörungsnarrativen innerhalb der Corona-Protestszene. Es stellt sich nun abschließend die Frage, warum dieses Milieu so anfällig für derartige Motive ist? Dazu einige Vorschläge:

Pia Lamberty und Katharina Nocun nennen drei Motive für Verschwörungsglaube:

Existentielle Motive (Streben nach Kontrolle und Sicherheit), soziale Motive (Streben nach einer positiven Wahrnehmung des eigenen Selbst und der Gruppe), epistemische Motive (Streben nach Verstehen und subjektiver Gewissheit). (Lamberty und Nocun 2021, S. 120)

Ein weiteres Motiv, das sich schon in Leo Löwenthals Skizze der faschistischen Agitatoren findet, ist die Selbst-Distinktion als „Wissende“. Sie passt sehr gut zum Bedürfnis gerade der Neuen Rechten, ein elitärer Kreis Eingeweihter zu sein. Wende ich zudem die Erkenntnis Löwenthals an, dass das „Selbstbild“ des Anhängers faschistischer Agitation vor allem ein ,umgekehrtes Feindbild“ darstellt, so ergeben alle Dämonisierungen, schiefen historischen Analogien und wüsten Spekulationen der Verschwörungsszene um so mehr Sinn (Löwenthal 1990, S. 124). Wer gegen das derart geballte „Böse“ kämpft, ist selbst eine Lichtgestalt. Das passt zum Befund von Lamberty und Nocun (2021, S. 121), dass „Menschen mit einem starken Bedürfnis nach Einzigartigkeit eher anfällig für Verschwörungsglauben“ sind.

Ich möchte hier nur kurz darauf hinweisen, dass in diesem Deutungsmuster den Aufdeckern der „Verschwörung“ ganz klassisch die Rolle des Katechon zukommt; einer theologischen Figur, deren Aufgabe es ist, den Antichristen aufzuhalten und der gerade im konservativ-revolutionären Denken etwa bei Carl Schmitt eine identitätsstiftende Rolle zukommt.

Die Leipziger Autoritarismusstudie weist auf die Verbindung zum magischen Denken hin. Dieses ist „eine normale Entwicklungsstufe der Kindheit“, doch

kann der Rückfall auf diese Wahrnehmungsart als Regression auf eine frühere Entwicklungsstufe verstanden werden: In diesem Fall reagieren Erwachsene auf Bedrohung und Stress, indem sie in eine Kontrollillusion flüchten (Decker und Brähler 2020: 300). 
Schließlich ist, in Anlehnung an meine eingangs vorgetragenen Überlegungen, in Verschwörungstheorien eine Verfallsform von Herrschaftssoziologie zu sehen: Hier ist das Motiv des autoritären Rebellen zu nennen, das sich bei Neurechten ebenso herauskristallisiert wie bei Alternativen und Esoterikern.

Darauf verweis auch die Leipziger Autoritarismusstudie, indem sie das Motiv der Rebellion gegen Schwäche und Illegitimität aufgreift.

„Der ,regressive Rebell“ ... befreit sich dabei zwar vielleicht von der Autorität etablierter politischer Institutionen und Akteure, eventuell jedoch nur, um sich in diesem Zuge anderen, besseren, weil stärker erscheinenden Autoritäten zu unterwerfen." (ebd.: 304)

Das können Eingeweihte, Naturheiler, alternative Medien oder auch der illiberale Staat der Neuen Rechten etc. sein. Die Rebellion gegen die liberale Demokratie speist sich also aus der Verachtung ihrer angeblichen Schwäche. Das würde zumindest erklären, warum z.B. die wesentlich strikteren Corona-Maßnahmen in Staaten wie Ungarn oder China kein Thema waren - Ungarns Orban wird weiterhin bewundert. Trotz des rebellischen und ,widerständigen“ Anspruchs der Proteste legt das Ergebnis der Leipziger Studie daher den Schluss nahe, dass der „Glaube an eine COVID-19-Verschwörung ... mit klassisch autoritären Dispositionen einher[geht]“ (ebd.: 304).

Nach all diesen Beobachtungen geben die in den Corona-Protesten kursierenden Verschwörungsnarrative also durchaus Anlass zur Sorge. Sie wirkten als „Radikalisierungsbeschleuniger“", trugen zur Abkapselung und Imprägnierung des Milieus bei und sorgten für eine tiefe Entfremdung gegenüber der liberalen Demokratie. Daher kann auch ich aus meiner Warte dem Fazit der Leipziger Autoritarismusstudie nur beipflichten: „Zu glauben, COVID-19 sei eine Verschwörung, ist ... nicht besonders demokratisch, rebellisch, unkonventionell oder friedlich und damit vor allem nicht per se anti-autoritär“ (ebd.: 305). Der Verschwörungsglaube in den Corona-Protesten und die Rede vom „Great Reset“ erweisen sich letztlich als ein weiteres Element einer als Freiheitskampf verkleideten autoritären Revolte.

\section{Literatur}

Amadeu Antonio Stiftung (Hrsg.). 2021. Down the Rabbit Hole. Verschwörungsideologien: Basiswissen und Handlungsstrategien.

Ballweg, Michael. 2021. „Wir entscheiden, wie wir leben - und das mit vereinten Kräften“. In Demokratischer Widerstand 40/2021, 11.

Benz, Wolfgang (Hrsg.). 2021. Querdenken. Protestbewegung zwischen Demokratieverachtung, Hass und Aufruhr.

Brenner, Peter J. 2021. „Tabula rasa. Bilder einer zerfallenden Gesellschaft“. In Tumult 2/2021, 10-14.

Bröckers, Mathias. 2002. Verschwörungen, Verschwörungstheorien und die Geheimnisse des 11.9.

Butter, Michael. 2018. „Nichts ist, wie es scheint. “ Über Verschwörungstheorien.

Decker, Oliver, und Elmar Brähler (Hrsg.). 2020. Leipziger Autoritarismusstudie 2020. Autoritäre Dynamiken: Alte Ressentiments - neue Radikalität.

Dorn, Christian. 2020. „Sie müssen den Gegner, den Feind, genau kennen!“‘. In Junge Freiheit 44/2020, 11.

Hoof, Thomas. 2021. „In zehn oder hundert Jahren“. In Sezession 100/2021, 30-37.

Institut für Soziologie der Universität Basel (Hrsg.). 2020. Politische Soziologie der Corona-Proteste. Grundauswertung v. 17.12.2020. 
Kießling, Simon. 2021. „Neue Normalität - faustisches Weltgefühl - Transhumanismus“. In Tumult 2/2021, 78-81.

Kleffner, Heike, und Matthais Meisner (Hrsg.). 2021. Fehlender Mindestabstand. Die Coronakrise und die Netzwerke der Demokratiefeinde.

Lamberty, Pia, und Katharina Nocun. 2021. Ein Brandbeschleuniger für Radikalisierung? Verschwörungserzählungen während der Covid-19-Pandemie. In Fehlender Mindestabstand. Die Coronakrise und die Netzwerke der Demokratiefeinde, Hrsg. Heike Kleffner, Matthias Meisner, 117-125.

Löwenthal, Leo. 1990. Falsche Propheten. Studien zum Autoritarismus 3.

Mies, Ullrich. 2021. „Transnationaler Staatsterror“. In Demokratischer Widerstand 36/2021, $1 \mathrm{ff}$.

Ministerium des Inneren des Landes Nordrhein-Westfalen. 2021. Sonderbericht zu Verschwörungsmythen und, Corona-Leugnern'. (Stand Mai 2021).

Österreichische Bundesstelle für Sektenfragen (Hrsg.). 2021. Das Phänomen Verschwörungstheorien in Zeiten der COVID-19-Pandemie. Bericht an die Bundesministerin für Frauen, Familie, Jugend und Integration. Wien.

Salzborn, Samuel. 2021. Verschwörungsmythen und Antisemitismus. Aus Politik und Zeitgeschichte (APuZ) 35/36:41-47. (Verschwörungstheorien).

Sommerfeld, Caroline. 2021. „Im Zugriff des Widersachers“. In Sezession 100/2021, 44-48.

Speit, Andreas. 2021. Verqueres Denken. Gefährliche Weltbilder in alternativen Milieus. 